Cronfa - Swansea University Open Access Repository

This is an author produced version of a paper published in :

Cortex

Cronfa URL for this paper:

http://cronfa.swan.ac.uk/Record/cronfa30949

\title{
Paper:
}

Towler, J. \& Eimer, M. (2015). Early stages of perceptual face processing are confined to the contralateral hemisphere: Evidence from the N170 component. Cortex, 64, 89-101.

http://dx.doi.org/10.1016/j.cortex.2014.09.013

This article is brought to you by Swansea University. Any person downloading material is agreeing to abide by the terms of the repository licence. Authors are personally responsible for adhering to publisher restrictions or conditions. When uploading content they are required to comply with their publisher agreement and the SHERPA RoMEO database to judge whether or not it is copyright safe to add this version of the paper to this repository. http://www.swansea.ac.uk/iss/researchsupport/cronfa-support/ 


\title{
Research report
}

\section{Early stages of perceptual face processing are confined to the contralateral hemisphere: Evidence from the N170 component}

\author{
John Towler* and Martin Eimer \\ Department of Psychological Sciences, Birkbeck College, University of London, London, UK
}

\section{A R T I C L E I N F O}

Article history:

Received 13 March 2014

Reviewed 19 June 2014

Revised 9 July 2014

Accepted 13 September 2014

Action editor Jason Barton

Published online 22 October 2014

Keywords:

Face perception

N170

Retinotopic

Category-selective

Competition

Ventral pathway

\begin{abstract}
A B S T R A C T
High-level visual object processing is often assumed to be largely position-independent. Here we demonstrate that when faces and non-face objects simultaneously appear in opposite visual hemifields, the face-sensitive N170 component of the event-related brain potential (ERP) is exclusively generated in the contralateral hemisphere. The effects of face inversion on N170 amplitudes and latencies also show strong contralateral biases. These results reveal that retinotopic biases in low-level visual cortex extend well into categoryselective high-level vision. We suggest that the contralateral organisation of facesensitive visual processing results from generic competitive interactions between hemispheres during the simultaneous perception of visual objects.
\end{abstract}

(c) 2014 Elsevier Ltd. All rights reserved.

\section{Introduction}

High-level object vision is often believed to depend upon representations which are independent of the retinal position of an object. This popular view is explicit in hierarchical models of the primate ventral visual stream (e.g., Ungerleider \& Mishkin, 1982). Such models assume that early stages of visual processing in striate and extrastriate occipital cortex represent simple features in a small spatially restricted region of the visual field, whereas complex object representations in inferior temporal cortex (IT) are largely position-invariant. While it is certainly true that receptive field size and the complexity of stimulus selectivity increases along the occipitotemporal visual pathway (e.g., Desimone \& Gross, 1979; Gross, RochaMiranda, \& Bender, 1972; Rousselet, Thorpe, \& Fabre-Thorpe, 2004; Tsao, Freiwald, Tootell, \& Livingstone, 2006), the question whether these changes are accompanied by a corresponding decrease in retinotopic biases of visual neurons has remained controversial (see Kravitz, Saleem, Baker, Ungerleider, \& Mishkin, 2013, for a review). For example, single-unit data have demonstrated a strong bias towards the

\footnotetext{
* Corresponding author. Department of Psychological Sciences, Birkbeck College, University of London, Malet Street, London WC1E 7HX, UK.

E-mail address: j.towler@bbk.ac.uk (J. Towler). http://dx.doi.org/10.1016/j.cortex.2014.09.013
} 0010-9452/@ 2014 Elsevier Ltd. All rights reserved. 
contralateral visual field in macaque anterior IT (Desimone \& Gross, 1979; Op De Beeck \& Vogels, 2000). While some human functional neuroimaging studies have suggested that highlevel visual object representations are not strongly affected by changes in retinotopic position (e.g., Grill-Spector et al., 1999), others found evidence for strong position-dependence (e.g., Kravitz, Kriegeskorte, \& Baker, 2010; Niemeier, Goltz, Kuchinad, Tweed, \& Vilis, 2005; see also Afraz \& Cavanagh, 2008, for the retinotopic nature of behavioural face adaptation effects). Hemond, Kanwisher, and Op De Beeck (2007) demonstrated a preference for contralateral stimuli in faceand object-selective regions. This preference was largest in primary visual cortex, and smaller but still reliably present in more anterior regions, such as the fusiform face area (FFA). The observation that this contralateral bias was present both for preferred and non-preferred visual categories (e.g., for nonface objects in face-selective areas; Hemond et al., 2007) suggests that it primarily reflects retinotopic input from lowerlevel visual areas that are not category-selective.

The limited temporal resolution of fMRI precludes direct insights into the emergence of contralateral biases in category-selective visual processing within the first $200 \mathrm{msec}$ after stimulus onset. The aim of the present study was to use event-related brain potentials (ERPs) to investigate whether early stages of category-selective processing in the human brain are position-dependent or position-invariant. We measured the face-sensitive N170 component, which is an enhanced negativity for faces as compared to non-face objects that is typically observed around $140-200 \mathrm{msec}$ after stimulus onset over posterior occipito-temporal electrode sites (e.g., Bentin, Allison, Puce, Perez, \& McCarthy, 1996). The N170 is assumed to reflect early visual-perceptual structural encoding stages that precede face recognition and identification (Eimer, 2000a). Source localisation studies have pointed to middle fusiform gyrus (FFA), inferior occipital gyrus (OFA), and the superior temporal sulcus (STS) as possible generators of the N170 (Bötzel, Schulze, \& Stodieck, 1995; Itier \& Taylor, 2004; Rossion, Joyce, Cottrell, \& Tarr, 2003; Watanabe, Kakigi, \& Puce, 2003). Intracranial recordings from pre-surgical patients have recently observed face-selective responses in the right OFA and FFA in the same time-window as the N170 component (Jonas et al., 2012; Parvizi et al., 2012; see also Horovitz, Rossion, Skudlarski, \& Gore, 2004; Sadeh, Podlipsky, Zhdanov, \& Yovel, 2010, for correlations between haemodynamic and ERP markers of face processing).

In addition to its generic face-sensitivity, the N170 is also affected by manipulations known to impair face recognition, such as contrast reversal (Itier \& Taylor, 2002), scrambling the locations of facial features (George, Evans, Fiori, Davidoff, \& Renault, 1996; Zion-Golumbic \& Bentin, 2007), and stimulus inversion (e.g., Bentin et al., 1996; Eimer, 2000b; Rossion et al., 1999; 2000). Relative to upright faces, the N170 to inverted faces is delayed and enhanced. The delay of the N170 in response to inverted faces suggests that the onset of facespecific processing is delayed when inversion alters the prototypical spatial relationships between face parts (Rossion et al., 2000). The inversion-induced increase of N170 amplitudes has been attributed to the increased difficulty of processing inverted faces (e.g., Rossion et al., 1999), or to the additional recruitment of populations of eye-selective neurons (Itier, Alain, Sedore, \& McIntosh, 2007) or populations of object-selective neurons (Rossion et al., 1999, 2000; Rosburg et al., 2010) by upside-down faces.

The aim of the current study was to find out if the N170 component shows a contralateral bias. Is the differential N170 response to faces versus non-face objects exclusively triggered over the contralateral hemisphere or is it also elicited on the ipsilateral side? Are the effects of face inversion on N170 amplitudes and latencies restricted to the contralateral side or are they unaffected by the retinal location of upright and inverted faces? These questions have not been systematically addressed, because face and non-face stimuli were presented at fixation in nearly all previous N170 studies. With foveal stimulus presentation, the $\mathrm{N} 170$ is elicited bilaterally, and is often more pronounced over the right hemisphere (see Eimer, 2011; Rossion \& Jacques, 2011, for reviews). In one earlier study from our lab (Eimer, 2000c), faces or non-faces (chairs) were presented unilaterally on the left or right of fixation. The N170 component was larger over the contralateral hemisphere, but was also present ipsilaterally (see also Rousselet, Husk, Bennett, \& Sekuler, 2005, for the presence of bilateral face-sensitive N170 components to single laterally presented faces and houses). While larger contralateral N170 amplitudes to faces presented in the left or right visual field (e.g., Eimer, 2000c; Jacques \& Rossion, 2004; Kovács, Zimmer, Volberg, Lavric, \& Rossion, 2013; see also Smith, Gosselin, \& Schyns, 2004, for evidence of larger N170 amplitudes triggered by the contralateral eye for centrally presented faces) may suggest that face-specific perceptual processing has a retinotopic bias, such findings need to be interpreted with caution. Because the N170 is a visual evoked ERP component, it is affected by basic physical stimulus features such as luminance, spatial frequency, and, importantly, retinal location (for a discussion of low-level stimulus properties and the face-sensitive N170 component, see Rossion \& Jacques, 2008). Visual evoked components to single visual stimuli on the left or right side tend to be generally larger over the contralateral hemisphere, regardless of the category of these stimuli (e.g., Clarke et al., 1994; Jeffreys \& Axford, 1972), and enhanced contralateral $\mathrm{N} 170$ amplitudes to unilateral faces may reflect this basic fact. For this methodological reason, it is preferable to use bilateral stimulus displays when investigating contralateral biases in category-selective perceptual face processing, as reflected by the $\mathrm{N} 170$ component.

There are also empirical reasons to assume that contralateral biases in category-specific object processing may differ for unilateral and bilateral visual displays. Single-unit studies of neurons in monkey temporal cortex (Chelazzi, Duncan, Miller, \& Desimone, 1998) have revealed that these neurons respond to the identity of single visual objects regardless of whether they are presented in the contralateral or ipsilateral hemifield. In contrast, when two or more objects appear simultaneously in opposite visual hemifields, these neurons respond selectively to the contralateral object, but remain largely unaffected by the identity of the object in the ipsilateral hemifield. The presence of a competing object in the other hemifield appears to be sufficient to eliminate stimulusspecific neural responses in the ipsilateral hemisphere, presumably by inhibiting category-specific input signals from contralateral to ipsilateral temporal cortex (see also Hornak, Duncan, \& Gaffan, 2002). If this is the case, contralateral 
biases in face-selective visual processing should be particularly pronounced for bilateral displays that contain a face on one side and a non-face object on the other.

Alternatively, it is possible that neural responses in faceselective visual areas to bilateral face/non-face displays represent a weighted average of the response of these areas when each stimulus is presented in isolation. Populations of face-selective neurons in occipito-temporal cortex have large receptive fields that include both contralateral and ipsilateral visual fields (e.g., Afraz \& Cavanagh, 2008; Eimer, 2000c; Jacques \& Rossion, 2004; Op De Beeck \& Vogels, 2000; Rolls \& Tovee, 1995; Tovee, Rolls, \& Azzopardi, 1994). When a preferred stimulus (a face) and a non-preferred stimulus (a house) are presented simultaneously within the receptive field of such neurons, the response to the pair of stimuli may be reduced relative to the response to the preferred stimulus alone (e.g., Kastner, De Weerd, Desimone, \& Ungerleider, 1998; Miller, Gochin, \& Gross, 1993; Reddy, Kanwisher, \& van Rullen, 2009). Such reductions of neural response rate caused by the addition of a non-preferred object have indeed been demonstrated for simple visual stimuli (oriented coloured lines) in area V4 of macaque visual cortex (Reynolds, Chelazzi, \& Desimone, 1999). If the same mechanisms also operate for complex visual stimuli at early category-selective processing stages, N170 components triggered by face-house pairs should be smaller than N170 components to bilateral arrays containing two faces, and this amplitude reduction should be observed both contralaterally and ipsilaterally.

In the present ERP study, we employed symmetrical bilateral displays. In Experiment 1, these displays could include two faces, a face on one side and a house on the other, or two houses. Here, the generic face sensitivity of the N170 component should be reflected by enhanced N170 amplitudes over the left and right hemisphere in response to displays with two faces as compared to displays with two houses. If the N170 was fully position-invariant, displays containing a face on the left and a house on the right (or vice versa) should also trigger bilateral N170 components relative to displays with bilateral houses. Due to the presence of a non-face stimulus, N170 components to face/house pairs might be smaller than the N170 to displays with bilateral faces. Alternatively, if the N170 was strictly position-dependent, larger N170 components should only be observed over the hemisphere contralateral to the visual field of a face. At ipsilateral electrodes, ERPs in the N170 time range should be indistinguishable from ERPs measured in response to bilateral houses.

\section{Experiment 1}

\subsection{Method}

\subsubsection{Participants}

Twelve paid participants with normal or corrected to normal vision were tested (five male, 21-36 years old, mean age: 25.4 years). All participants were right handed.

\subsubsection{Stimuli and procedure}

Stimulus displays consisted of bilateral naturalistic photographic images of faces (taken from Ekman \& Friesen, 1976) and houses. The stimulus set consisted of ten different faces with neutral expression and ten different houses. All stimuli subtended a visual angle of approximately $2.8^{\circ} \times 4.0^{\circ}$. Stimuli were presented using E-prime software (Psychology Software Tools, Pittsburgh, PA) at a viewing distance of $100 \mathrm{~cm}$. On each trial, two images were presented simultaneously to the left and right of fixation at a horizontal eccentricity of approximately $2.5^{\circ}$, measured relative to the centre of each image. Each bilateral stimulus array was presented for $200 \mathrm{msec}$, and the intertrial interval was $1000 \mathrm{msec}$. A fixation cross was continuously present at the centre of the screen throughout each experimental block. Four different array types were presented with equal probability and in random order. Displays could contain one face on the left side and a different face on the right (FF), two different houses on the left and right side $(\mathrm{HH})$, a face on the left and a house on the right $(\mathrm{FH})$, or a left-side house and a right-side face (HF). On a small subset of trials, a target (a red box around one of the two stimuli) was present. Participants were instructed to maintain central fixation, and to press a response key with their right hand whenever a target box was present. Four blocks of 88 trials were run. Each block contained 80 nontarget trials (20 trials for each of the four display types) and eight target trials.

\subsubsection{EEG recording and analysis}

Electroencephalogram (EEG) was DC-recorded with a Brainamps DC amplifier with an upper cutoff frequency of $40 \mathrm{~Hz}$ and a sampling rate of $500 \mathrm{~Hz}$ from $23 \mathrm{Ag}-\mathrm{AgCl}$ electrodes mounted in an elastic cap according to the extended 10 to 20 system at scalp sites Fpz, F7, F3, Fz, F4, F8, FC5, FC6, T7, C3, Cz, C4, T8, CP5, CP6, P7, P3, Pz, P4, P8, PO7, PO8, and Oz. No further filters were applied after EEG acquisition. Horizontal eye movements (HEOG) were measured from two electrodes placed at the outer canthus of each eye. The right earlobe was recorded as an additional channel. All electrodes were referenced to the left earlobe during recording and were later digitally transformed to a common average reference including the right earlobe. Impedances of all electrodes were kept below $5 \mathrm{k} \Omega$. All off-line analyses of EEG data were conducted with Brain Vision Analyser software. The EEG was epoched into 400-msec segments from $100 \mathrm{msec}$ before stimulus onset to $300 \mathrm{msec}$ after stimulus onset. Trials with saccades (voltage exceeding $\pm 30 \mu \mathrm{V}$ in the HEOG channel), eye blinks (exceeding $\pm 60 \mu \mathrm{V}$ at Fpz), or muscular artifacts (exceeding $\pm 80 \mu \mathrm{V}$ at any other electrode) were excluded from analysis. Only nontarget trials (i.e., trials without the red target box) were included for EEG analysis. The N170 component was quantified on the basis of ERP mean amplitudes obtained in the 150-190 msec post-stimulus interval at P7/P8. The P1 component was quantified based on ERP mean amplitudes measured during the $80-120 \mathrm{msec}$ post-stimulus interval at $\mathrm{P} 7 / \mathrm{P} 8$.

\subsection{Results}

\subsubsection{Behavioural performance}

Participants correctly detected $95 \%$ of all target items. Mean reaction time (RT) on target trials was $585 \mathrm{msec}$. There were no False Alarms on non-target trials. 
A

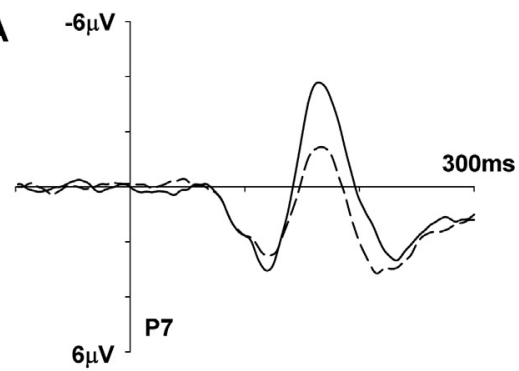

B

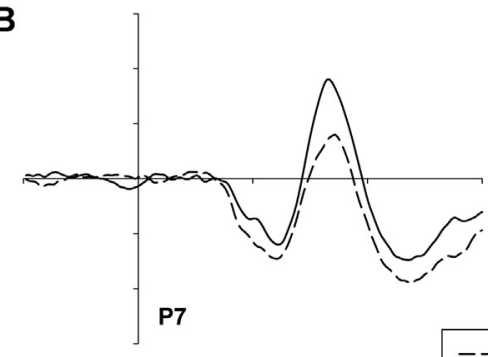

- Ipsilateral Face/Contralateral House
Contralateral Face/lpsilateral House
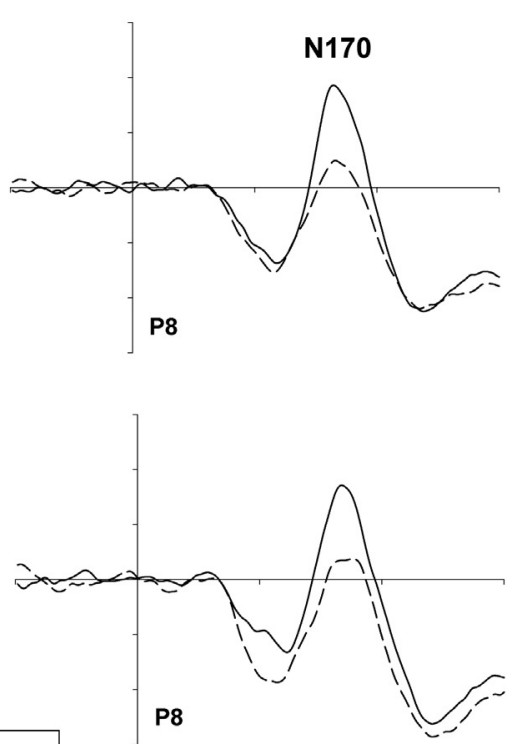

P8
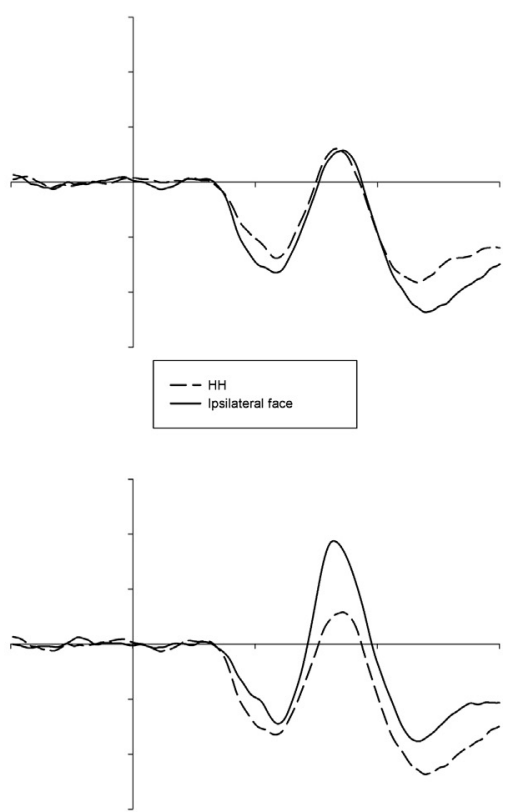

- $\mathrm{FF}$

D

C

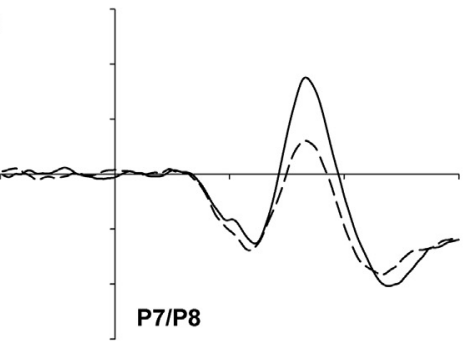

- - Con Contralateral face

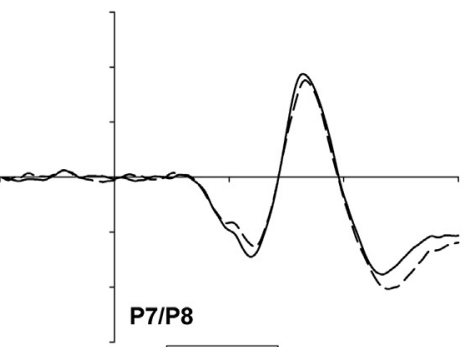

- ${ }_{\text {FF }}$

$\mathbf{E}$

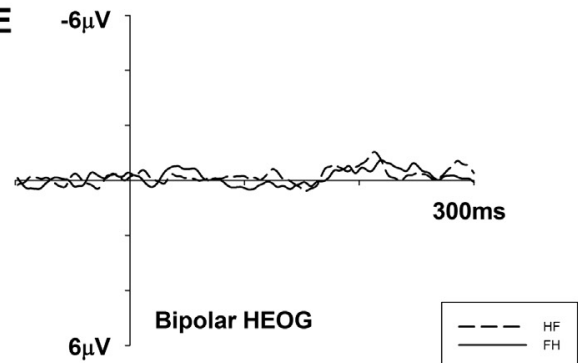

Fig. 1 - Grand-averaged ERPs elicited in in Experiment 1 at lateral temporo-occipital electrodes P7 (left hemisphere) and P8 (right hemisphere) in the 300 msec interval after stimulus onset. (A) ERPs elicited in response to displays with two faces on the left and right side (FF, solid lines), and two houses on the left and right (HH, dashed lines). (B) ERPs elicited at P7 and P8 in response to displays with a face on one side and a house on the other, shown separately for trials where the face appears contralateral (solid lines) and ipsilateral (dashed lines) to the recording electrode. (C) ERPs elicited in response to displays with two houses (HH, dashed lines, collapsed across P7 and P8), compared with ERPs triggered contralateral to the face in 


\subsubsection{N170 component}

Fig. 1A shows N170 components triggered in response to displays with bilateral faces or bilateral houses (FF and $\mathrm{HH}$ displays) at electrodes P7 and P8. There was a main effect of display type, $F(1,11)=18.51, p=.001, \eta_{p}^{2}=.63$, as ERP mean amplitudes in the N170 time window (150-190 msec poststimulus) were larger for $\mathrm{FF}$ relative to $\mathrm{HH}$ displays, thus confirming the presence of the face-sensitive N170 component. This face-sensitive N170 was not significantly different in size over the left and right hemisphere, as demonstrated by the absence of a display type $\times$ hemisphere interaction, $F<1$, nor was there a main effect of hemisphere on general N170 amplitudes, $F<1$. Fig. 1B shows ERPs in response to displays with a face and a house on opposite sides (FH and HF trials) at P7 and P8. They are displayed separately for contralateral faces (HF displays at P7, and FH displays at F8, dashed lines) and ipsilateral faces (FH displays at P7, and HF displays at F8, solid lines). The N170 was clearly larger over the contralateral hemisphere. To assess this formally, N170 mean amplitudes at $\mathrm{P} 7 / 8$ in response to $\mathrm{FH}$ and $\mathrm{HF}$ displays were compared in an ANOVA with the factors laterality (electrode contralateral us ipsilateral to the face) and hemisphere. A significant effect of laterality was observed, $F(1,11)=17.61, p=.001, \eta_{p}^{2}=.62$, confirming the contralateral dominance of the N170. There was no significant laterality $\times$ hemisphere interaction, $F<1$.

To determine whether the face-sensitive N170 was exclusively triggered contralaterally, ERPs measured to $\mathrm{FH}$ and HF displays were compared to ERPs in response to displays with bilateral houses $(\mathrm{HH})$. Fig. 1C shows ERPs elicited contralateral or ipsilateral to the side of the face in $\mathrm{FH}$ and HF displays (solid lines) and on $\mathrm{HH}$ trials (dashed lines, collapsed across P7/8). Enhanced N170 amplitudes to displays containing a lateral face were present over the contralateral hemisphere, $t(11)=4.1, p<.005$; but this effect was completely abolished ipsilaterally, $t<.1$. To further confirm the contralaterality of the N170 component, ERPs triggered by face/house displays were also compared to ERPs in response to displays with bilateral faces (FF). Fig. 1D shows ERPs elicited contralateral or ipsilateral to the side of the face in $\mathrm{FH}$ and HF displays (solid lines) and on FF trials (dashed lines, collapsed across P7/8). The contralateral N170 component for bilateral face/house displays was not significantly different in size to the N170 measured in response to bilateral faces, $t<1$.4. In contrast, N170 mean amplitudes over the ipsilateral hemisphere were strongly attenuated relative to bilateral face displays, $\mathrm{t}(11)=4.7, p>.001$.

The exclusively contralateral nature of generic N170 face selectivity, and the strong contralateral dominance of inversion-induced N170 amplitude modulations are illustrated in the topographical maps in Fig. 2. The top panels show scalp distributions measured in Experiment 1 during the N170 time window (150-190 msec post-stimulus) in response to displays with two faces (left), a face on the right and a house on the left (middle), and a face on the right and a house on the left (right). These maps show difference amplitudes relative to the non-face baseline condition (bilateral houses). Faceselective N170 amplitude enhancements are triggered bilaterally for displays with two faces, and are exclusively confined to the contralateral hemisphere when faces was accompanied by a house on the other side.

N170 amplitudes are often largest at electrode sites P7 and P8 but are also present on other lateral posterior electrodes (see the top panels of Fig. 2 for scalp maps showing the lateral posterior distribution of the face-selective N170). To confirm that the contralaterality of the N170 face selectivity was not confined to electrode sites $\mathrm{P} 7 / \mathrm{P} 8$, further analyses were performed on the more posterior electrode pair PO7/PO8. These analyses replicated the pattern of results found for $\mathrm{P} 7 / \mathrm{P} 8$. N170 components were larger for displays containing two faces as compared to two houses, $F(1,11)=10.65, p=.008$, $\eta_{p}^{2}=.49$. Importantly, N170 amplitudes were larger contralateral to the location of the face in $\mathrm{FH}$ and HF displays, $F(1,11)=14.18, p=.003, \eta_{p}^{2}=.56$. Follow up t-tests also confirmed that N170 components were significantly present contralateral to the location of faces when compared to $\mathrm{HH}$ displays, $t(11)=3.01, p=.012$, but were not significantly present ipsilaterally, $t<1.8$.

In summary, these results demonstrate that when two objects are presented simultaneously in the left and right visual field, the generic face-sensitivity of the N170 (i.e., N170 amplitude enhancements for faces relative to non-face objects) is entirely confined to the contralateral hemisphere. When a face and a house appeared together on opposite sides, the N170 component contralateral to the face was identical to the N170 triggered by bilateral face pairs, whereas the ipsilateral N170 was indistinguishable from the N170 elicited by bilateral house pairs. In other words, a differential brain response to faces versus houses was triggered contralaterally, but was completely eliminated over the ipsilateral hemisphere.

\subsubsection{Eye movements}

The strongly contralateral N170 component observed in Experiment 1 could in principle be linked to eye movements towards the side of the face in $\mathrm{FH}$ and HF displays. Although the N170 component is triggered before eye movements are typically initiated ( $<200 \mathrm{msec}$ post-stimulus), it is important to confirm that there was no large systematic deviations of gaze direction during the first $300 \mathrm{msec}$ after stimulus onset. Fig. 1E shows HEOG waveforms measured during this interval in response to $\mathrm{FH}$ and $\mathrm{HF}$ displays. Although HEOG channels are unlikely to detect microsaccades, any large tendency for the eyes to move towards the location of face stimuli in these displays should have been reflected by HEOG deviations of

\footnotetext{
face/house displays (left panel, solid line), and ERPs triggered ipsilateral to the face (right panel, solid line). (D) ERPs elicited in response to displays with two faces (FF, dashed lines, collapsed across P7 and P8), compared with ERPs triggered contralateral to the face in face/house displays (left panel, solid line), and ERPs triggered ipsilateral to the face (right panel, solid line). (E) Horizontal EOG waveforms elicited in the $300 \mathrm{msec}$ interval after stimulus onset in response to FH and $\mathrm{HF}$ displays. Negative (upward-going) deflections represent eye movements towards the right side, and positive deflections represent leftward eye movements.
} 


\section{A}
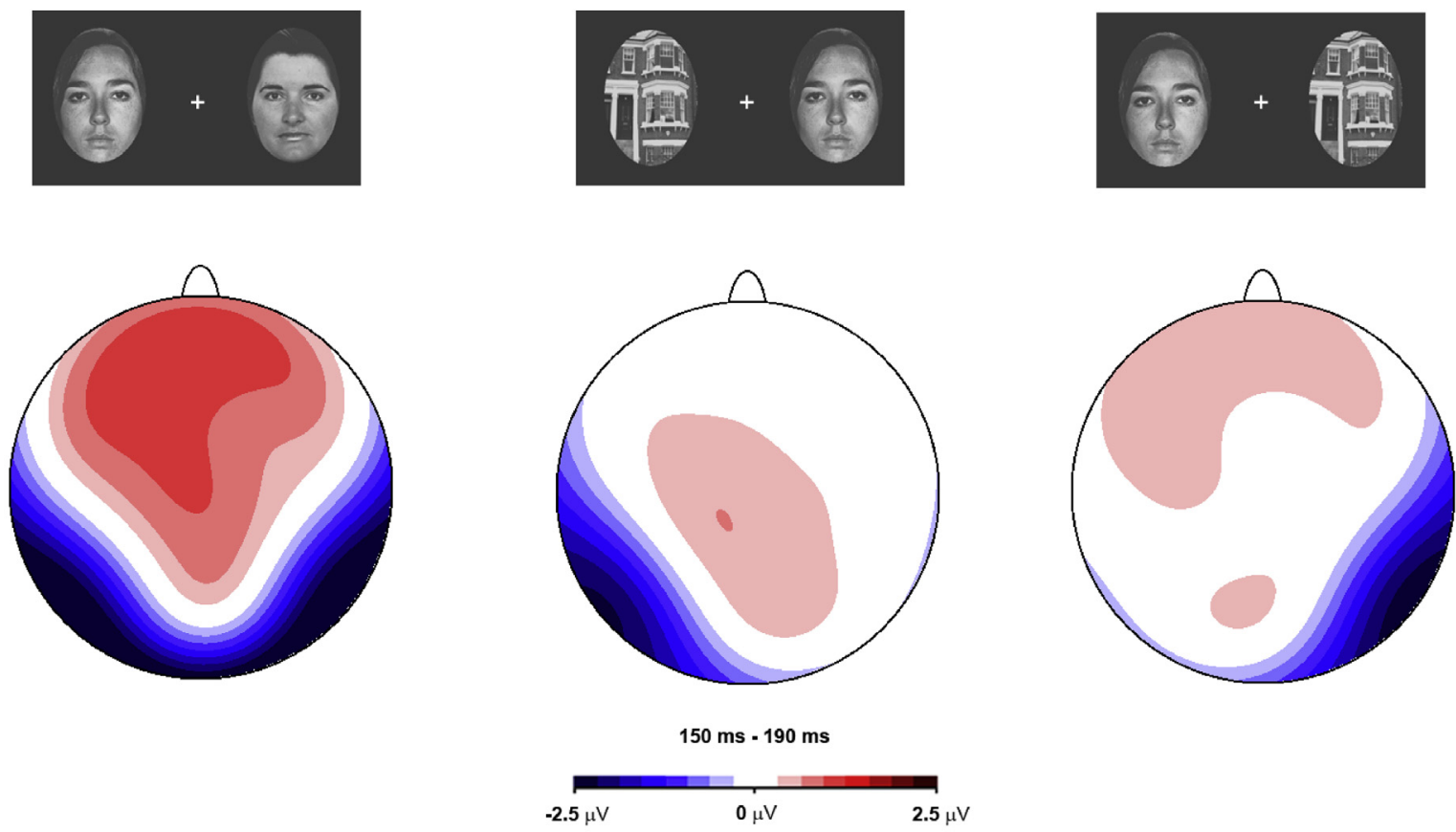

B
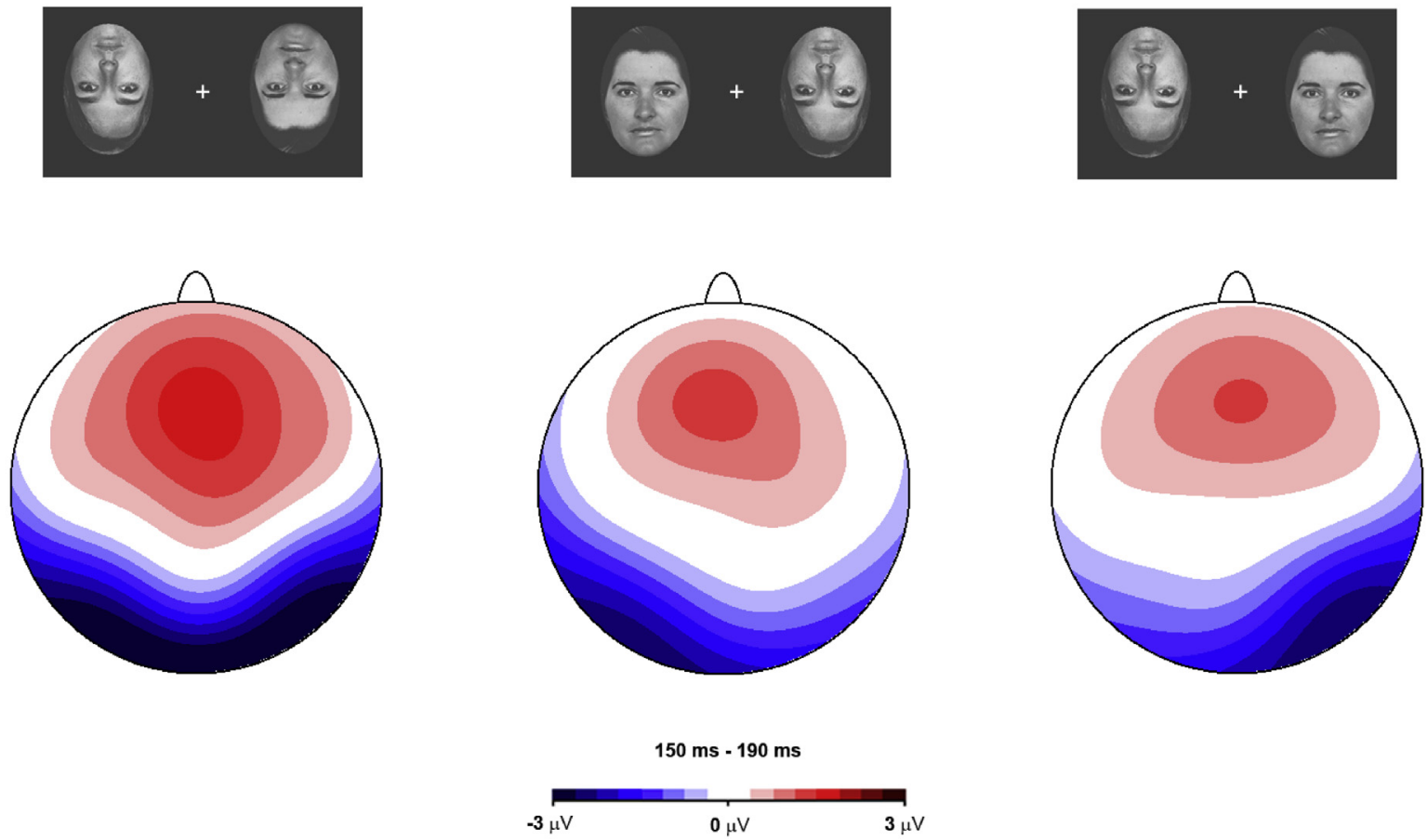

Fig. 2 - Topographic maps of ERP difference amplitudes measured during the N170 time window (150-190 msec poststimulus). (A) Difference amplitudes measured in Experiment 1 relative to the non-face baseline condition with two houses on either side. Bilateral N170 components were triggered in response to bilateral faces (left panel). For unilateral faces on the right or left (middle and right panels), the N170 was elicited exclusively over the contralateral hemisphere. (B) Difference amplitudes measured in Experiment 2 relative to the baseline condition with two upright faces on either side. Bilateral inversion-induced N170 amplitude enhancements were elicited in response to bilateral inverted faces (left panel). For unilateral inverted faces on the right or left that were accompanied by an upright face on the other side (middle and right panels), face inversion effects on N170 amplitudes showed a strong contralateral bias. 
opposite polarity in these HEOG waveforms. As can be seen from Fig. 1E, there was no evidence for any such systematic deviation of gaze, confirming that participants maintained central fixation.

\subsubsection{P1 component}

The visually evoked P1 component that precedes the N170 was quantified on the basis of ERP mean amplitudes obtained at $\mathrm{P} 7 / 8$ in the $80-120 \mathrm{msec}$ post-stimulus time window. As can be seen in Fig. 1A, there were no P1 amplitude difference between $\mathrm{FF}$ and $\mathrm{HH}$ trials, $\mathrm{F}<1$, indicating that the P1 is not sensitive to the difference between faces and houses. However, a comparison of $\mathrm{P} 1$ components to $\mathrm{FH}$ and HF displays revealed a significant main effect of contralaterality, $F(1,11)=23.24, p<.001, \eta_{p}^{2}=.68$. P1 components were reliably larger contralateral to the side where the house was presented in these displays (Fig. 1B), and this contralateral bias did not differ between the two hemispheres, $F<3$.2. The significance of these P1 results will be briefly discussed in the General Discussion.

\section{Experiment 2}

This second experiment investigated whether the effects of face inversion on the N170 component are also exclusively confined to the contralateral hemisphere. Procedures were the same as in Experiment 1, except that houses were replaced by inverted faces. Displays contained two upright faces, two inverted faces, or an upright and an inverted face on opposite sides. Relative to displays with two upright faces, two inverted faces should trigger typical bilateral N170 face inversion effects (enhanced and delayed N170 components for inverted versus upright faces). If these effects mirror the pattern observed in Experiment 1 for the generic facesensitivity of the N170 component, they should be elicited exclusively over the hemisphere contralateral to an inverted face that is presented together with an upright face on the other side, and be entirely absent ipsilaterally.

\subsection{Method}

\subsubsection{Participants}

Twelve paid participants were tested in Experiment 2 (five male, 24-39 years old, mean age: 29 years). None of the participants in Experiment 2 took part in Experiment 1. All participants were right handed.

\subsubsection{Stimuli, procedure, EEG recording, and analysis} Procedures were similar to Experiment 1, except that upright and inverted faces were used as stimuli, and no house images were shown. Ten inverted face images were created by rotating the ten upright face photographs by $180^{\circ}$. There were four equiprobable and randomly presented display types upright faces on both sides (UU), inverted faces on both sides (II), an upright face on the left and an inverted face on the right (UI), or an inverted face on the left and an upright face on the right (IU). In addition to N170 mean amplitudes which were measured in the 150-190 msec latency window, N170 peak latencies were also computed within the 150-190 msec latency window. P1 mean amplitudes were measured between 80 and $120 \mathrm{msec}$ after stimulus onset over electrodes P7 and P8.

\subsection{Results}

\subsubsection{Behavioural performance}

Target detection accuracy was $95 \%$ and mean target RT was $604 \mathrm{msec}$. There were no False Alarms on non-target trials.

\subsubsection{N170 component}

Fig. 3A shows N170 components triggered at P7 and P8 in response to displays with two upright faces or two inverted faces (UU and II displays) at electrodes P7 and P8. N170 amplitudes were larger for bilateral inverted faces, $F(1,11)=37.5$, $p<.001, \eta_{p}^{2}=.77$, and this N170 face inversion effect did not differ between hemispheres, $F<1.1$. It appears as though N170 components tended to be slightly larger over the right hemisphere than over the left hemisphere irrespective of the orientation of the face stimulus. The lack of a main effect of hemisphere, however, did not confirm this observation, $F<1$. There was also the expected delay of N170 peak latencies for bilateral inverted as compared to upright faces, [172 msec us 167 msec; $F(1,11)=7.1, p<.03, \eta_{p}^{2}=.39$ ], which did not interact with hemisphere, $F<1$. Fig. 3B shows ERPs in response to displays with an upright and an inverted face on opposite sides at P7 and P8. ERPs are displayed separately for contralateral inverted faces (UI displays at P7, and IU displays at F8, dashed lines) and ipsilateral inverted faces (IU displays at P7, and UI displays at F8, solid lines). Enhanced N170 amplitudes were elicited contralaterally to the visual field of the inverted face. This was confirmed by a significant effect of laterality, electrode contralateral versus ipsilateral to the inverted face; $F(1,11)=15.1, p<.005, \eta_{p}^{2}=.579$, that did not interact with hemisphere, $F<1$. N170 peak latencies also tended to be delayed at contralateral versus ipsilateral electrodes (172 msec us $169 \mathrm{msec}$ ), but the effect of laterality on N170 latency only approached significance, $F(1,11)=3.8, p=.074$, $\eta_{p}^{2}=.26$.

To find out whether N170 face inversion effects were entirely confined to the contralateral hemisphere, ERPs in response to displays with an upright and inverted face on opposite sides were compared to ERPs elicited by displays with bilateral upright faces. Fig. 3C shows N170 components triggered contralateral (left) or ipsilateral (right) to the side of the inverted face and ERPs to displays with two upright faces (solid lines, collapsed across P7/8). As expected, the presence of an inverted face triggered larger N170 amplitudes over the contralateral hemisphere, $t(11)=4.45, p<.001$. A small but reliable N170 enhancement was also observed ipsilateral to the inverted face, $t(11)=3.28, p<.01$. The contralateral inversion-induced N170 amplitude enhancement was reliably larger than the corresponding ipsilateral effect, $t(11)=3.89$, $p<.005$. The usual N170 peak latency delay for inverted faces was reliably present contralaterally [172 msec us $167 \mathrm{msec}$, $t(11)=4.1, p<.005]$. Over the ipsilateral hemisphere, there was a tendency in the same direction which only approached significance [169 msec us $167 \mathrm{msec}, \mathrm{t}(11)=1.97, p=.074]$.

As a final test of the contralaterality of N170 face inversion effects, we compared ERPs to displays with an upright and 
A

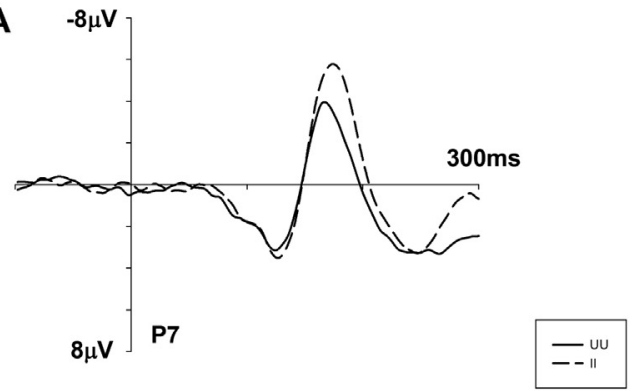

B

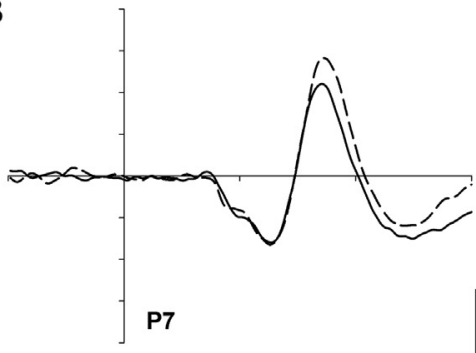

C

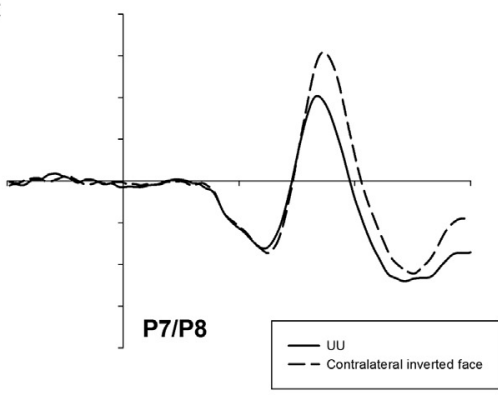

D

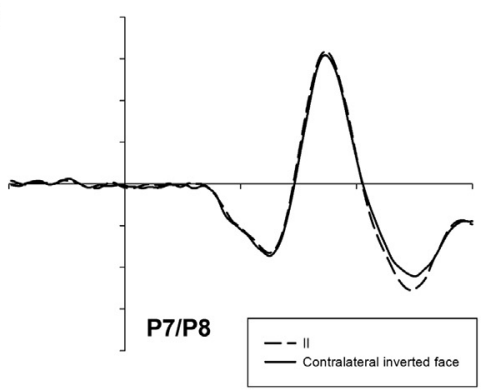

N170

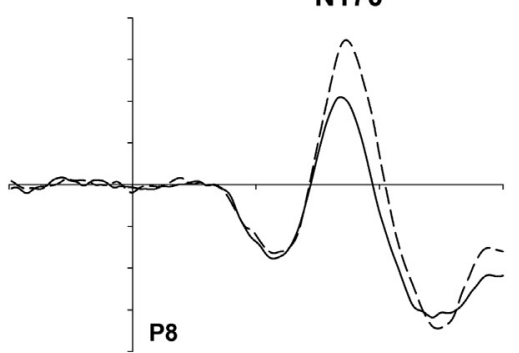

P8
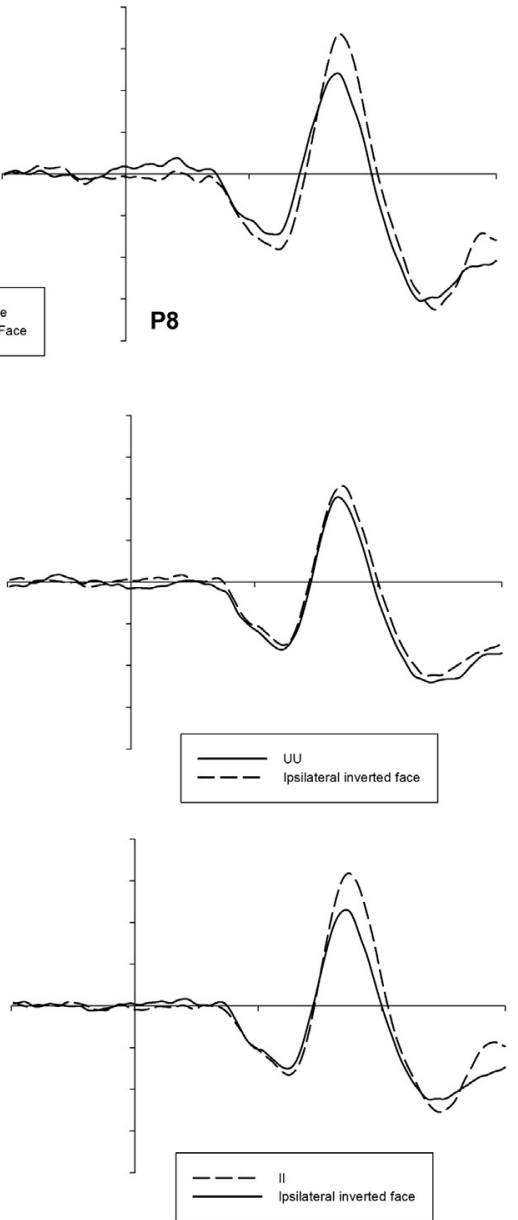

E

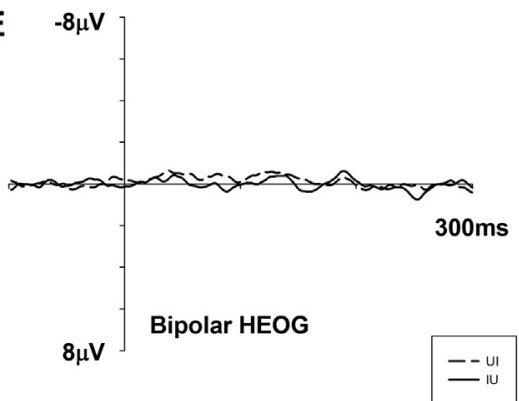

Fig. 3 - Grand-averaged ERPs elicited in in Experiment 2 at lateral temporo-occipital electrodes P7 (left hemisphere) and P8 (right hemisphere) in the $\mathbf{3 0 0} \mathrm{msec}$ interval after stimulus onset. (A) ERPs elicited in response to displays with two upright faces on the left and right side (UU, solid lines), and two inverted faces on the left and right (II, dashed lines). (B) ERPs elicited at P7 and P8 in response to displays with an upright face on one side and an inverted face on the other, shown separately for trials where the inverted face appears contralateral (dashed lines) and ipsilateral (solid lines) to the recording electrode. (C) ERPs elicited in response to displays with two upright faces (UU, solid lines, collapsed across P7 and P8), compared with 
inverted face on opposite sides and ERPs to displays with two inverted faces (Fig. 3D). At electrodes ipsilateral to the side of an inverted face (right panel), N170 amplitudes were much smaller relative to displays with bilateral inverted faces, $t(11)=5.86, p<.001$, reflecting the strong reduction of the N170 face inversion effect over the ipsilateral hemisphere. The corresponding N170 peak latency difference (172 msec us $169 \mathrm{msec}$ for displays with bilateral us unilateral inverted faces) was not reliable $(t<1.2)$. There was a small tendency for larger N170 amplitudes in response to bilateral inverted faces relative to the contralateral N170 (Fig. 3D, left panel), presumably reflecting the additional contribution of residual ipsilateral N170 inversion effects on bilateral trials. However, this difference only approached significance, $t(11)=1.94$, $p=.074$.

Fig. 2 (bottom panels) shows the pattern of inversioninduced N170 amplitude enhancements observed in Experiment 2. These scalp distribution maps show difference amplitudes for displays with two inverted faces (left), with an inverted face on the right and an upright face on the left (middle), and with an inverted face on the left and an upright face on the right (right), relative to baseline displays with two upright faces. Inverted face pairs triggered bilateral N170 face inversion effects. When inverted faces were accompanied by upright faces on the other side, these effects were much larger over the contralateral hemisphere.

In order to confirm that the strong contralaterality of N170 face inversion effects was not confined to electrode sites P7/P8, analogous analyses were performed on the more posterior lateral electrode pair PO7/PO8. These analyses confirmed the presence of face inversion effects at these additional electrode sites (II vs UU displays), $F(1,11)=55.89$, $p<.001, \eta_{p}^{2}=.84$. Importantly, the comparison of UI and IU displays revealed a main effect of laterality, $F(1,11)=22.39$, $p=.001, \eta_{p}^{2}=.67$, with larger N170 components at electrodes contralateral to the location of the inverted face in these displays. In summary, the effects of face inversion on N170 amplitudes and latencies showed a strong contralateral bias. When an upright and an inverted face appeared together on opposite sides, N170 enhancements and delays observed contralateral to the inverted face were indistinguishable from the effects found for bilateral inverted faces, while ipsilateral N170 face inversion effects were strongly attenuated. The fact that these ipsilateral effects were reduced but not completely eliminated will be discussed below.

\subsubsection{Eye movements}

As in Experiment 1, it is important to confirm that there was no large systematic deviation of gaze direction during the first $300 \mathrm{msec}$ after stimulus onset. Fig. 3E shows HEOG waveforms measured during this interval in response to UI and IU displays. Any large tendency for the eyes to move towards the location of either the upright or inverted face stimuli in these displays should have been reflected by HEOG deviations of opposite polarity in these HEOG waveforms. As can be seen from Fig. 3E, there was no evidence for any such systematic deviation of gaze, confirming that participants maintained central fixation.

\subsubsection{P1 component}

P1 amplitudes showed no differential modulation to upright versus inverted face, and this was the case irrespective of the location of these faces in the visual field (Fig. 3). There was no P1 amplitude difference between UU and II displays, $F<1.7$. The comparison of UI and IU displays obtained no effect of laterality, $F<1.8$, demonstrating that there were no P1 amplitude differences between electrodes contralateral versus ipsilateral to the location of an inverted face.

\section{Discussion}

The question whether category-specific visual processing in occipitotemporal cortex retains the retinotopic organisation of lower-level visual areas or is position-invariant is still controversial. The current study provides new evidence for strong contralateral biases in perceptual face processing that are reflected by the face-sensitive N170 component. Experiment 1 demonstrated that the generic face-sensitivity of the N170 (i.e., N170 amplitude enhancements for faces relative to non-face objects) is entirely confined to the contralateral hemisphere. Experiment 2 showed that the effects of face inversion on the N170 component (larger and delayed N170 components for inverted versus upright faces) also have a strong contralateral bias. The observation that differential N170 responses to faces versus houses were completely abolished over the ipsilateral hemisphere is consistent with previous findings by Chelazzi et al. (1998). These authors demonstrated that when two or more competing objects are simultaneously present in both hemifields, object-selective neural responses in macaque temporal cortex are confined to the contralateral hemisphere, and are absent ipsilaterally. This lack of any stimulus-specific ipsilateral neural activity suggests that the presence of a competing object in the other visual field inhibits the transmission of information about stimulus identity from contralateral to ipsilateral temporal cortex. The exclusively contralateral nature of the N170 component observed in Experiment 1 is fully in line with this account. It is important to note that different results will be observed under conditions where a single visual object appears in isolation in the left or right visual field. This was shown in a previous experiment from our lab (Eimer, 2000c),

ERPs triggered contralateral to the inverted face in upright/inverted face displays (left panel, dashed line), and ERPs triggered ipsilateral to the inverted face (right panel, dashed line). (D) ERPs elicited in response to displays with two inverted faces (II, solid lines, collapsed across P7 and P8), compared with ERPs triggered contralateral to the inverted face in upright/inverted face displays (left panel, dashed line), and ERPs triggered ipsilateral to the inverted face (right panel, dashed line). (E) Horizontal EOG waveforms elicited in the $300 \mathrm{msec}$ interval after stimulus onset in response to IU and UI displays. Negative (upward-going) deflections represent eye movements towards the right side, and positive deflections represent leftward eye movements. 
where N170 amplitude enhancements to unilaterally presented faces versus chairs were larger contralaterally, but were also present at ipsilateral electrodes (for additional evidence for large ipsilateral face-sensitive N170 components with single laterally presented stimuli see Rousselet et al., 2005). Along very similar lines, Chelazzi et al. (1998) demonstrated that identity-selective neural responses are triggered both in contralateral and ipsilateral macaque temporal cortex when single-item unilateral displays are employed, although these responses still tend to be larger contralaterally. The presence of a competing stimulus in the opposite visual field appears to be a necessary condition for inhibiting the transmission of identity-sensitive information between hemispheres.

An alternative possibility was that N170 amplitudes to face/house pairs might be reduced at both contralateral and ipsilateral electrodes relative to N170 components to displays with two faces, because the presence of a non-preferred stimulus may have caused a general inhibition of neural activity in face-selective visual cortex (e.g., Reddy et al., 2009; Reynolds et al., 1999). Experiment 1 found no evidence for a reduction of contralateral $\mathrm{N} 170$ amplitudes in response to displays with face/house pairs (FH and HF trials) as compared to displays with bilateral faces (FF trials). As can be seen in Fig. 1D, contralateral N170 amplitudes were virtually identical to N170 amplitudes on FF trials, while the ipsilateral N170 was strongly attenuated. These observations suggest that when there is simultaneous temporal competition between two visual objects, the processing of category-selective information operates independently in the two cerebral hemispheres.

The absence of N170 components ipsilateral to the location of the face on $\mathrm{FH}$ and HF trials observed in Experiment 1 demonstrates that the processing of category-selective information is suppressed in the ipsilateral hemisphere. However, one could argue that this type of processing might only be delayed ipsilaterally, and may emerge beyond the N170 time window. As can be seen in Fig. 1C (right panel), there is no evidence for an enhanced ipsilateral negativity for $\mathrm{FH} / \mathrm{HF}$ trials relative to $\mathrm{HH}$ trials at longer post-stimulus latencies. If anything, an enhanced positivity can be observed between 200 and 300 msec after stimulus onset over electrodes P7/P8.

The strong contralateral bias for N170 face inversion effects observed in Experiment 2 provides further evidence for the position-dependence of face-specific visual processing. If category-selective signals are not transmitted between hemispheres when a competing object is simultaneously present on the other side, as suggested by the absence of an ipsilateral face-sensitive N170 response in Experiment 1, no effects of face inversion on N170 amplitudes and latencies should be elicited ipsilaterally. In fact, there was a small but significant N170 amplitude increase ipsilateral to an inverted face in upright/inverted face displays relative to displays with two upright faces, and a tendency towards an inversioninduced ipsilateral N170 latency delay (Fig. 3C). If information about the category of a visual object (face us house) is not accessible to the ipsilateral hemisphere, how can the ipsilateral N170 show residual signs of sensitivity to face inversion? It is possible that the small ipsilateral N170 face inversion effects observed in Experiment 2 do not reflect face-specific processing, but are instead a generic response to non- canonical views of objects. Support for this idea comes from a study by Itier, Latinus, and Taylor (2006), who found that stimulus inversion delayed N170 components not just for faces, but also for non-face objects like houses or chairs. The same study also observed enhanced N170 amplitudes for inverted as compared to upright houses (see also Eimer, 2000b; Nemrodov, Anderson, Preston, \& Itier, 2014, for analogous findings), suggesting that inversion effects on N170 amplitudes may also not be as face-specific as usually thought (see also Busey \& Vanderkolk, 2005, for evidence that N170 inversion effects for non-face objects are modulated by visual expertise). Along similar lines, a recent rapid adaptation study from our lab (Eimer, Kiss, \& Nicholas, 2010, Experiment 2) found that adaptation effects on the N170 component to inverted faces show both category-selectivity and orientationsensitivity: N170 amplitudes were attenuated when inverted faces were preceded by faces versus non-face objects (houses). They were further attenuated when inverted faces were preceded by inverted objects (faces or houses) versus upright objects. The presence of generic face-unspecific orientationinduced N170 adaptation effects suggests that categoryunspecific neural populations tuned to non-canonical object orientations may contribute to N170 face inversion effects. The possibility that the small inversion-induced ipsilateral N170 amplitude enhancement observed in the present study might be linked to face-unselective neural responses to inverted objects can be tested in future studies by assessing inversion effects for bilateral pairs of non-face objects.

In the present study, N170 components to faces were not significantly larger over the right hemisphere. A righthemisphere dominance of the N170 is commonly observed in studies which have compared the processing of faces to other classes of visual stimuli such as non-face objects or words (e.g., Rossion et al., 2003). It is possible that righthemisphere biases in early face processing may be restricted to situations where a single face is presented in isolation. Alternatively, this bias might be eliminated whenever another competing object is simultaneously present in the opposite visual field. These possibilities will need to be systematically investigated in future research.

In Experiment 1, ERP differences between contralateral and ipsilateral electrodes in response to face/house displays were already present for the visually evoked P1 component (see Fig. 1). P1 components did not differ between displays with bilateral faces and bilateral houses, thus demonstrating that in contrast to the $\mathrm{N} 170$, the $\mathrm{P} 1$ is not generally sensitive to faces versus non-face objects. However, ERP amplitudes in the P1 time window were more negative contralateral to the face in FH and HF displays, and this resulted in larger P1 amplitudes at electrodes contralateral to the side of the house in these displays. This lateralised P1 modulation (which was absent in Experiment 2 for displays that contained an upright and an inverted face) was unexpected, and its neural basis remains unclear. It is possible that this effect is linked to a lateralised response to faces versus non-face objects that is triggered at short latencies in contralateral low-level visual areas that is triggered specifically when both types of objects appear simultaneously in opposite visual fields. If this is correct, such early low-level visual responses may contribute to the contralateral dominance of face-specific perceptual 
processing that emerges at a later category-selective stage of visual processing that is reflected by the N170 component. The involvement of such lower-level (or category-unspecific) neural populations in producing contralateral neural responses to complex objects such as faces is consistent with category-unspecific contralateral biases demonstrated previously with neuroimaging (e.g., Hemond et al., 2007).

Overall, the current study has demonstrated that perceptual stages of face-selective processing are strongly positiondependent in the sense that they are appear to be exclusively confined to the contralateral hemisphere. While it is generally accepted that early visual areas contain retinotopic maps of the contralateral visual field (e.g., Wandell, Dumoulin, \& Brewer, 2007), the question whether higher-level processing in object-selective occipitotemporal cortex show a similar degree of position-dependence remains controversial (see Kravitz et al., 2013, for review). The current results demonstrate strong contralateral biases for the differential processing of faces versus non-faces, and of inverted versus upright faces. The exclusively contralateral nature of generic N170 face sensitivity (Experiment 1) and the strong contralateral dominance of $\mathrm{N} 170$ face inversion effects (Experiment 2) show that when two stimuli or more appear simultaneously in both visual fields, populations of neurons in face-selective visual areas are primarily or perhaps even exclusively responsive to face-specific information from the contralateral hemifield.

It is unlikely that the hemisphere ipsilateral to the spatial position of complex visual objects is entirely blind to their presence when they are accompanied by one or more objects on the opposite side. For example, populations of visual neurons in occipito-temporal cortex are known to respond to the presence of objects in a category-unspecific fashion (e.g., Desimone, Albright, Gross, \& Bruce, 1984; Malach et al., 1995). It is also possible that some small degree of category-selective neural activity is present in the ipsilateral hemisphere. ERP measures depend upon large populations of neurons which are orientated in a uniform direction to produce electrical currents that are observable on the scalp (e.g., Luck, 2005), and may therefore not be able to pick up small category-sensitive neural activity modulations that are generated ipisilaterally. In line with this possibility, single cell recordings of macaque visual cortex have shown that there may be some residual object-selective neural activity in ipsilateral neurons even in the presence of an additional stimulus (Chelazzi et al., 1998). However, and in line with the current N170 results in human participants, the activity of these neurons is almost entirely supressed by the addition of a second stimulus in the opposite visual field. In the present study, we demonstrate that at large-scale levels of neural activity there is a strong and consistent contralateral organisation of category-selective neural responses at early perceptual stages of high-level vision.

The functional consequences of this contralateral dominance (or ipsilateral suppression) of category-selective information remain to be determined. One intriguing possibility is that each cerebral hemisphere is able to effectively process one complex visual object at a time. Working memory capacity appears to be much more limited for faces and other complex objects (Curby \& Gauthier, 2007; Eng, Chen, \& Jiang, 2005) relative to simple visual features and conjunctions (e.g., Luck \& Vogel, 1997; Vogel, Woodman, \& Luck, 2001). Increasing the duration of the memory display (and allowing for eye movements during encoding) increases working memory capacity for complex objects but not for simple features (Curby \& Gauthier, 2007; Eng et al., 2005). Together with the current findings, these results suggest that capacity limitations in the encoding and maintenance of visual objects are due to competitive interactions between hemispheres during category-selective perceptual processing.

Our conclusion that the higher-level processing of visual object categories such as faces is strongly and perhaps even exclusively confined to the contralateral hemisphere only applies to situations where there are competing objects in both hemifields, and not to conditions where a single visual object appears in an otherwise empty visual field. However, competition between hemispheres is clearly a common phenomenon in everyday vision, as our visual world nearly always contains multiple objects in both visual fields. Therefore, the contralateral dominance of high-level visual object processing demonstrated by our results is likely to be the rule rather than the exception for real-world vision.

\section{Notes}

The authors would like to thank Brad Duchaine for comments on an earlier version of the manuscript. Conflict of interest: None declared.

\section{Acknowledgements}

This work was supported by a grant (ES/K002457/1) from the Economic and Social Research Council (ESRC), UK.

\section{R E F E R E N C E S}

Afraz, S. R., \& Cavanagh, P. (2008). Retinotopy of the face aftereffect. Vision Research, 48(1), 42-54.

Bentin, S., Allison, T., Puce, A., Perez, E., \& McCarthy, G. (1996) Electrophysiological studies of face perception in humans. Journal of Cognitive Neuroscience, 8(6), 551-565.

Bötzel, K., Schulze, S., \& Stodieck, S. R. (1995). Scalp topography and analysis of intracranial sources of face-evoked potentials. Experimental Brain Research, 104(1), 135-143.

Busey, T. A., \& Vanderkolk, J. R. (2005). Behavioral and electrophysiological evidence for configural processing in fingerprint experts. Vision Research, 45(4), 431-448.

Chelazzi, L., Duncan, J., Miller, E. K., \& Desimone, R. (1998). Responses of neurons in inferior temporal cortex during memory-guided visual search. Journal of Neurophysiology, 80(6), $2918-2940$

Clark, V. P., Fan, S., \& Hillyard, S. A. (1994). Identification of early visual evoked potential generators by retinotopic and topographic analyses. Human Brain Mapping, 2(3), 170-187.

Curby, K. M., \& Gauthier, I. (2007). A visual short-term memory advantage for faces. Psychonomic Bulletin \& Review, 14(4), $620-628$.

Desimone, R., Albright, T. D., Gross, C. G., \& Bruce, C. (1984). Stimulus-selective properties of inferior temporal neurons in the macaque. The Journal of Neuroscience, 4(8), 2051-2062. 
Desimone, R., \& Gross, C. G. (1979). Visual areas in the temporal cortex of the macaque. Brain Research, 178(2), 363-380.

Eimer, M. (2000a). Event-related brain potentials distinguish processing stages involved in face perception and recognition. Clinical Neurophysiology, 111(4), 694-705.

Eimer, M. (2000b). Effects of face inversion on the structural encoding and recognition of faces. Evidence from eventrelated brain potentials. Cognitive Brain Research, 10(1), 145-158.

Eimer, M. (2000c). Attentional modulations of event-related brain potentials sensitive to faces. Cognitive Neuropsychology, 17(1-3), 103-116.

Eimer, M. (2011). The face-sensitive N170 component of the event-related brain potential. In A. J. Calder (Ed.), The Oxford handbook of face perception (pp. 329-344). Oxford: University Press.

Eimer, M., Kiss, M., \& Nicholas, S. (2010). Response profile of the face-sensitive N170 component: a rapid adaptation study. Cerebral Cortex, 20(10), 2442-2452.

Ekman, P., \& Friesen, W. V. (1976). Measuring facial movement. Environmental Psychology and Nonverbal Behavior, 1(1), 56-75.

Eng, H. Y., Chen, D., \& Jiang, Y. (2005). Visual working memory for simple and complex visual stimuli. Psychonomic Bulletin \& Review, 12(6), 1127-1133.

George, N., Evans, J., Fiori, N., Davidoff, J., \& Renault, B. (1996). Brain events related to normal and moderately scrambled faces. Cognitive Brain Research, 4(2), 65-76.

Grill-Spector, K., Kushnir, T., Edelman, S., Avidan, G., Itzchak, Y., \& Malach, R. (1999). Differential processing of objects under various viewing conditions in the human lateral occipital complex. Neuron, 24(1), 187-203.

Gross, C. G., Rocha-Miranda, C. E., \& Bender, D. B. (1972). Visual properties of neurons in inferotemporal cortex of the Macaque. Journal of Neurophysiology, 35(1), 96.

Hemond, C. C., Kanwisher, N. G., \& de Beeck, H. P. O. (2007). A preference for contralateral stimuli in human object-and faceselective cortex. PLoS One, 2(6), e574.

Hornak, J., Duncan, J., \& Gaffan, D. (2002). The role of the vertical meridian in visual memory for objects. Neuropsychologia, 40(12), 1873-1880.

Horovitz, S. G., Rossion, B., Skudlarski, P., \& Gore, J. C. (2004). Parametric design and correlational analyses help integrating fMRI and electrophysiological data during face processing. NeuroImage, 22(4), 1587-1595.

Itier, R. J., \& Taylor, M. J. (2002). Inversion and contrast polarity reversal affect both encoding and recognition processes of unfamiliar faces: a repetition study using ERPs. NeuroImage, 15(2), 353-372.

Itier, R. J., \& Taylor, M. J. (2004). N170 or N1? Spatiotemporal differences between object and face processing using ERPs. Cerebral Cortex, 14(2), 132-142.

Itier, R. J., Alain, C., Sedore, K., \& McIntosh, A. R. (2007). Early face processing specificity: it's in the eyes! Journal of Cognitive Neuroscience, 19(11), 1815-1826.

Itier, R. J., Latinus, M., \& Taylor, M. J. (2006). Face, eye and object early processing: what is the face specificity? NeuroImage, 29(2), 667-676.

Jacques, C., \& Rossion, B. (2004). Concurrent processing reveals competition between visual representations of faces. NeuroReport, 15(15), 2417-2421.

Jeffreys, D. A., \& Axford, J. G. (1972). Source locations of patternspecific components of human visual evoked potentials. I. Component of striate cortical origin. Experimental Brain Research, 16(1), 1-21.

Jonas, J., Descoins, M., Koessler, L., Colnat-Coulbois, S., Sauvée, M., Guye, M., et al. (2012). Focal electrical intracerebral stimulation of a face-sensitive area causes transient prosopagnosia. Neuroscience, 222, 281-288.
Kastner, S., De Weerd, P., Desimone, R., \& Ungerleider, L. G. (1998). Mechanisms of directed attention in the human extrastriate cortex as revealed by functional MRI. Science, 282(5386), $108-111$.

Kovács, G., Zimmer, M., Volberg, G., Lavric, I., \& Rossion, B. (2013). Electrophysiological correlates of visual adaptation and sensory competition. Neuropsychologia, 51(8), 1488-1496.

Kravitz, D. J., Kriegeskorte, N., \& Baker, C. I. (2010). High-level visual object representations are constrained by position. Cerebral Cortex, 20(12), 2916-2925.

Kravitz, D. J., Saleem, K. S., Baker, C. I., Ungerleider, L. G., \& Mishkin, M. (2013). The ventral visual pathway: an expanded neural framework for the processing of object quality. Trends in Cognitive Sciences, 17(1), 26-49.

Luck, S. J. (2005). An introduction to the event-related potential technique. MIT press.

Luck, S. J., \& Vogel, E. K. (1997). The capacity of visual working memory for features and conjunctions. Nature, 390(6657), 279-281.

Malach, R., Reppas, J. B., Benson, R. R., Kwong, K. K., Jiang, H., Kennedy, W. A., et al. (1995). Object-related activity revealed by functional magnetic resonance imaging in human occipital cortex. Proceedings of the National Academy of Sciences, 92(18), 8135-8139.

Miller, E. K., Gochin, P. M., \& Gross, C. G. (1993). Suppression of visual responses of neurons in inferior temporal cortex of the awake macaque by addition of a second stimulus. Brain Research, 616(1), 25-29.

Niemeier, M., Goltz, H. C., Kuchinad, A., Tweed, D. B., \& Vilis, T. (2005). A contralateral preference in the lateral occipital area: sensory and attentional mechanisms. Cerebral Cortex, 15(3), 325-331.

Nemrodov, D., Anderson, T., Preston, F. F., \& Itier, R. J. (2014). Early sensitivity for eyes within faces: a new neuronal account of holistic and featural processing. NeuroImage, 97, 81-94.

Op De Beeck, H., \& Vogels, R. (2000). Spatial sensitivity of macaque inferior temporal neurons. Journal of Comparative Neurology, 426(4), 505-518.

Parvizi, J., Jacques, C., Foster, B. L., Withoft, N., Rangarajan, V., Weiner, K. S., et al. (2012). Electrical stimulation of human fusiform face-selective regions distorts face perception. The Journal of Neuroscience, 32(43), 14915-14920.

Reddy, L., Kanwisher, N. G., \& VanRullen, R. (2009). Attention and biased competition in multi-voxel object representations. Proceedings of the National Academy of Sciences, 106(50), 21447-21452.

Reynolds, J. H., Chelazzi, L., \& Desimone, R. (1999). Competitive mechanisms subserve attention in macaque areas V2 and V4. The Journal of Neuroscience, 19(5), 1736-1753.

Rolls, E. T., \& Tovee, M. J. (1995). The responses of single neurons in the temporal visual cortical areas of the macaque when more than one stimulus is present in the receptive field. Experimental Brain Research, 103(3), 409-420.

Rosburg, T., Ludowig, E., Dümpelmann, M., Alba-Ferrara, L., Urbach, H., \& Elger, C. E. (2010). The effect of face inversion on intracranial and scalp recordings of event-related potentials. Psychophysiology, 47(1), 147-157.

Rossion, B., \& Jacques, C. (2008). Does physical interstimulus variance account for early electrophysiological face sensitive responses in the human brain? Ten lessons on the N170. NeuroImage, 39(4), 1959-1979.

Rossion, B., \& Jacques, C. (2011). The N170: understanding the time-course of face perception in the human brain. In S. Luck, \& E. Kappenman (Eds.), The Oxford handbook of ERP components (pp. 115-142). Oxford: University Press.

Rossion, B., Delvenne, J. F., Debatisse, D., Goffaux, V., Bruyer, R., Crommelinck, M., et al. (1999). Spatio-temporal localization of 
the face inversion effect: an event-related potentials study. Biological Psychology, 50(3), 173-189.

Rossion, B., Gauthier, I., Tarr, M. J., Despland, P., Bruyer, R., Linotte, S., et al. (2000). The N170 occipito-temporal component is delayed and enhanced to inverted faces but not to inverted objects: an electrophysiological account of facespecific processes in the human brain. NeuroReport, 11(1), 69.

Rossion, B., Joyce, C. A., Cottrell, G. W., \& Tarr, M. J. (2003). Early lateralization and orientation tuning for face, word, and object processing in the visual cortex. NeuroImage, 20(3), 1609-1624.

Rousselet, G. A., Husk, J. S., Bennett, P. J., \& Sekuler, A. B. (2005). Spatial scaling factors explain eccentricity effects on face ERPs. Journal of Vision, 5(10), 1.

Rousselet, G. A., Thorpe, S. J., \& Fabre-Thorpe, M. (2004). How parallel is visual processing in the ventral pathway? Trends in Cognitive Sciences, 8(8), 363-370.

Sadeh, B., Podlipsky, I., Zhdanov, A., \& Yovel, G. (2010). Eventrelated potential and functional MRI measures of faceselectivity are highly correlated: a simultaneous ERP-fMRI investigation. Human Brain Mapping, 31(10), 1490-1501.

Smith, M. L., Gosselin, F., \& Schyns, P. G. (2004). Receptive fields for flexible face categorizations. Psychological Science, 15(11), 753-761.
Tovee, M. J., Rolls, E. T., \& Azzopardi, P. (1994). Translation invariance in the responses to faces of single neurons in the temporal visual cortical areas of the alert macaque. Journal of Neurophysiology, 72(3), 1049-1060.

Tsao, D. Y., Freiwald, W. A., Tootell, R. B., \& Livingstone, M. S. (2006). A cortical region consisting entirely of face-selective cells. Science, 311(5761), 670-674.

Ungerleider, L. G., \& Mishkin, M. (1982). Two cortical visual systems. Analysis of Visual Behavior, 549-586.

Vogel, E. K., Woodman, G. F., \& Luck, S. J. (2001). Storage of features, conjunctions, and objects in visual working memory. Journal of Experimental Psychology: Human Perception and Performance, 27(1), 92-114.

Wandell, B. A., Dumoulin, S. O., \& Brewer, A. A. (2007). Visual field maps in human cortex. Neuron, 56(2), 366-383.

Watanabe, S., Kakigi, R., \& Puce, A. (2003). The spatiotemporal dynamics of the face inversion effect: a magneto-and electroencephalographic study. Neuroscience, 116(3), 879-895.

Zion-Golumbic, E., \& Bentin, S. (2007). Dissociated neural mechanisms for face detection and configural encoding: evidence from N170 and induced gamma-band oscillation effects. Cerebral Cortex, 17(8), 1741-1749. 